\title{
Pericardial effusion and constriction as an initial presentation of acute t-cell lymphoblastic lymphoma
}

\author{
Angela Rao MD, Phumpattra Chariyawong MD, Teerapat Nantsupawat MD, \\ Irfan Warraich MD, Nandini Nair MD
}

\begin{abstract}
Acute T-cell lymphoblastic lymphoma is a rare subtype of non-Hodgkin's lymphoma accounting for $2 \%$ of adult non-Hodgkin's lymphomas. Cardiac involvement in non-Hodgkin's lymphoma is usually a late manifestation, and pericardial effusion as an initial presentation is rare. We report a case of acute T-cell lymphoblastic lymphoma in a patient who initially presented with pericardial effusion. Diagnosis was difficult and challenging, as an infectious etiology was first suspected. The patient presented with pericardial effusion without other common clinical manifestations of lymphoma, such as fever, night sweats, and weight loss. He was treated for constrictive pericarditis due to having positive Coxsackie $B$ serology but had no improvement with treatment. The pathology results of the pericardium after pericardiectomy reported fibrosis with a reactive lymphohistiocyte infiltrate. After CT scan revealed a large mediastinal mass, a lymph node biopsy was performed, and T-cell lymphoblastic lymphoma was confirmed. A detailed evaluation to search for occult malignancy should be considered in patients who have persistent pericarditis that is unresponsive to anti-inflammatory therapy and in those who present with a new, large pericardial effusion or cardiac tamponade.
\end{abstract}

Keywords: T-cell lymphoblastic lymphoma, constrictive pericarditis, pericardial effusion

\section{INTRODUCTION}

T-cell lymphoblastic lymphoma is a rare subtype of non-Hodgkin's lymphoma accounting for $2 \%$ of adult non-Hodgkin's lymphomas. ${ }^{1,2}$ The incidence is approximately 3 cases per $1,000,000$ people per year with a male predominance. ${ }^{3}$ Common clinical manifestations include fever, malaise, night sweats, weight loss, and painless lymphadenopathy. A mediastinal mass occurs in $50-75 \%$ of patients and may lead to superior vena cava syndrome, tracheal obstruction, pericardial effusion, and pleural effusion. However, cardiac involvement in non-Hodgkin's lymphoma is usually a late manifestation. ${ }^{4}$ Pericardial effusion and

Corresponding author: Angela Rao

Contact Information: AngelaRao104@gmail.com

DOI: $10.12746 /$ swrccc.v7i28.541 constriction as an initial presentation are rare. The diagnosis can be challenging due to difficulty to distinguish between malignant cells and reactive lymphoid cells. ${ }^{5,6}$ We report a case of acute T-cell lymphoblastic lymphoma in a patient who first presented with pericardial effusion and negative pathology of pericardial tissue. The diagnosis was subsequently confirmed by lymph node biopsy.

\section{CASE}

A 40-year-old man presented to emergency room with 2 weeks of pleuritic chest pain and shortness of breath. Physical examination showed distant heart sounds and pulsus paradoxus. There was no hepatosplenomegaly or peripheral lymphadenopathy. Complete blood counts upon admission showed a white blood cell count of $19,400 / \mu \mathrm{L}$ with neutrophil 
predominance, a hemoglobin of $13.4 \mathrm{gm} / \mathrm{dL}$, and a platelet count of $471,000 / \mu \mathrm{L}$ with no atypical platelets. He had a total protein of $7.1 \mathrm{gm} / \mathrm{dL}$, albumin of $3.8 \mathrm{~g} / \mathrm{dL}$, AST of $14 \mathrm{U} / \mathrm{L}, \mathrm{ALT}$ of $15 \mathrm{U} / \mathrm{L}$, and creatinine of $1.1 \mathrm{mg} / \mathrm{dL}$. The PTT was 32.9 seconds, and the PT was slightly high at 17.4 seconds. Other pertinent laboratory results included a calcium of $8.8 \mathrm{mg} / \mathrm{dL}$ [normal range $8.8-10.5 \mathrm{mg} / \mathrm{dL}$ ] and positive Coxsackie virus $B$ antibody. Echocardiography revealed moderate to large pericardial effusion with early tamponade physiology. Pericardiocentesis yielded approximately $550 \mathrm{~mL}$ of serosanguinous fluid. Pericardial fluid analysis showed WBC of $1,413 / \mathrm{mm}^{3}$ with $76 \%$ neutrophils and $24 \%$ lymphocytes. Cytologic study of the pericardial fluid was negative for neoplastic cells.

The patient was treated for viral pericarditis and sent home with colchicine and indomethacin; however, his symptoms persisted, and he developed bilateral pleural effusions after 4 weeks of colchicine and indomethacin and an additional 4 weeks of prednisone and furosemide. Follow-up echocardiography identified ventricular interdependence with respiration, inferior vena cava plethora, and abnormal lateral mitral annular tissue Doppler with medial annular sparing, consistent with constrictive pericarditis. Right heart catheterization (RHC) demonstrated dip and plateau of right atrial pressure waveform and discordant of RV/LV simultaneous pressure recording, confirming constrictive pericarditis as a diagnosis. During the RHC, he was unable to tolerate the supine position, which prompted a $2^{\text {nd }}$ admission to the hospital. Cardiothoracic surgery performed a pericardiectomy and drained both pleural effusions. Pericardial tissue pathology showed fibrotic tissue with lymphohistiocytic infiltrate, favoring a reactive process. The pleural effusion continued to drain per chest tube, and an indwelling pleural catheter was placed. The patient subsequently developed atrial fibrillation and atrial flutter postoperatively requiring cardioversion, and right sided heart failure secondary to atrial fibrillation developed. He was discharged after he converted to normal sinus rhythm and was hemodynamically stable and was instructed to follow up with cardiology as an outpatient.

He was admitted a $3^{\text {rd }}$ time, 5 months after his initial admission, due to repeat episodes of atrial flutter requiring ablation. Cavotricuspid isthmus ablation was repeated, and the patient was converted to normal sinus rhythm. The following $\mathrm{RHC}$ reported no findings of constriction, indicating successful pericardial stripping by cardiothoracic surgery; however, right ventricular stroke work index indicated severe right ventricular failure. Due to the lack of clinical improvement, computed tomography (CT) of chest was done. This scan of the chest revealed a large, ill-defined soft tissue mass infiltrating the mediastinum and bilateral axillary adenopathy consistent with malignancy (Figure 1). Excisional lymph node biopsy with immunohistochemistry was positive for CD3 (Figure 2), CD5 (Figure 3), CD7, TdT (Figure 4) and CD79a (Figure 5), consistent with $\mathrm{T}$ cell precursor lymphoblastic lymphoma. Flow cytology was done from the pleural effusion site and showed atypical B-cells with CD 45, CD 5/19, and CD 79a positive, and no light chain restriction detected. His left axillary biopsy was reviewed at the Mayo Clinic in Minnesota and revealed T-cell precursor acute lymphoblastic leukemia/lymphoma (ETP T-ALL) due to absence of CD1a and presence of CD33 surface

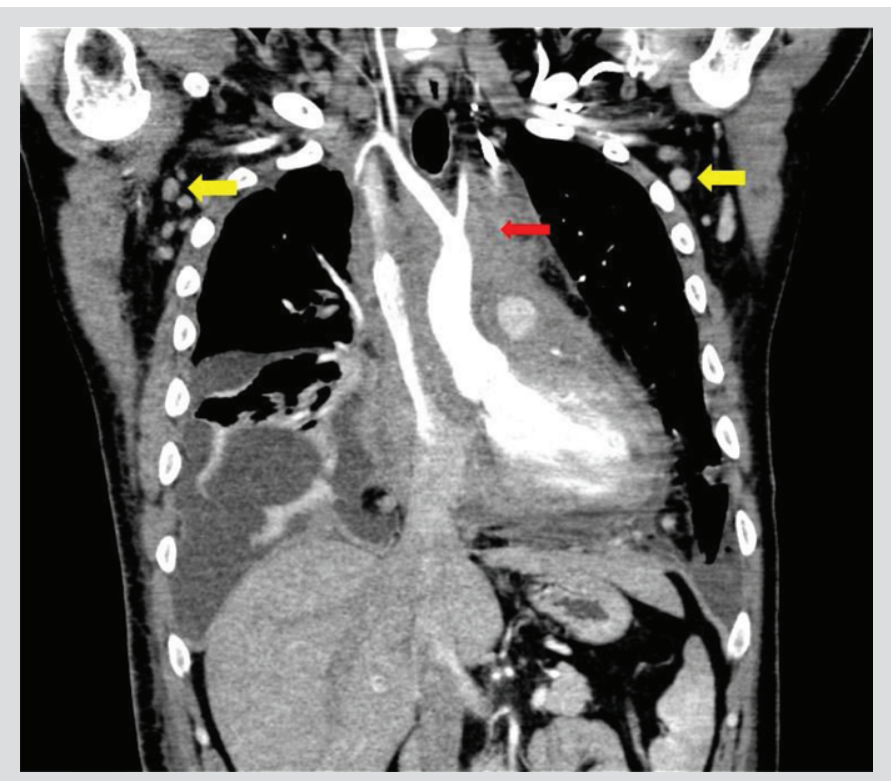

Figure 1. CT Chest.

Large ill-defined soft tissue mass infiltrating the mediastinum (thin red arrow). Bilateral axillary adenopathy (thick yellow arrow). 


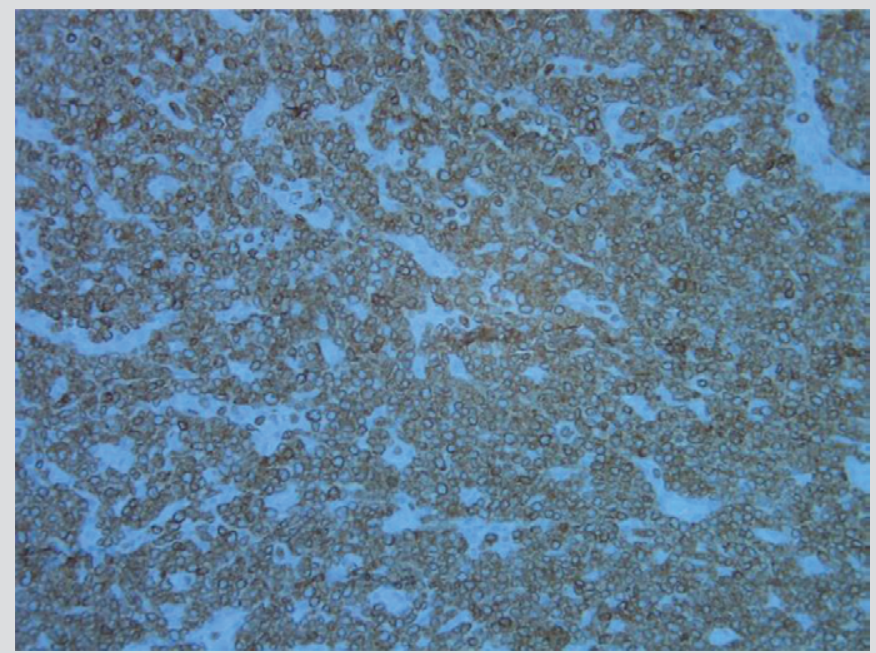

Figure 2. CD3.

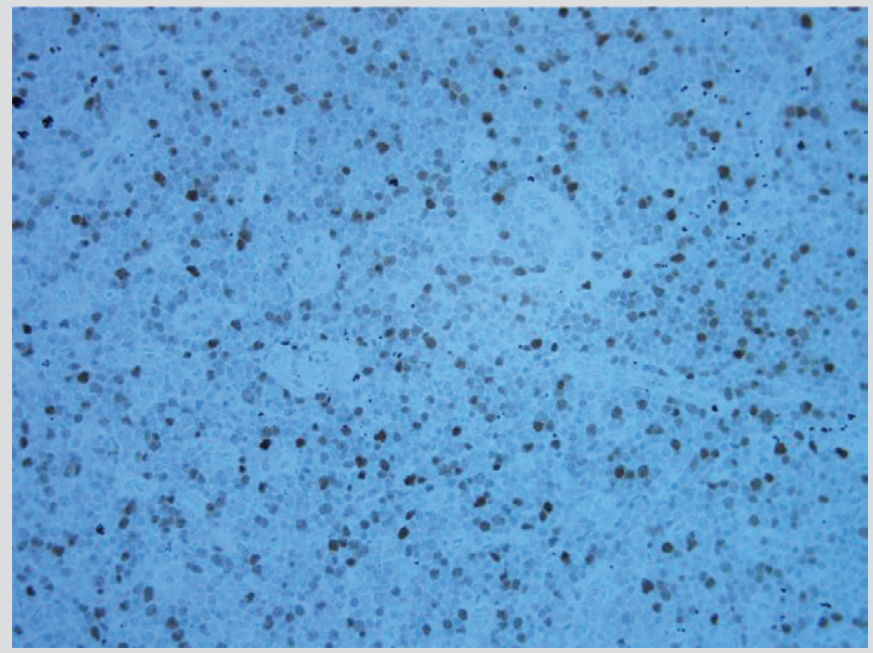

Figure 4. TdT.

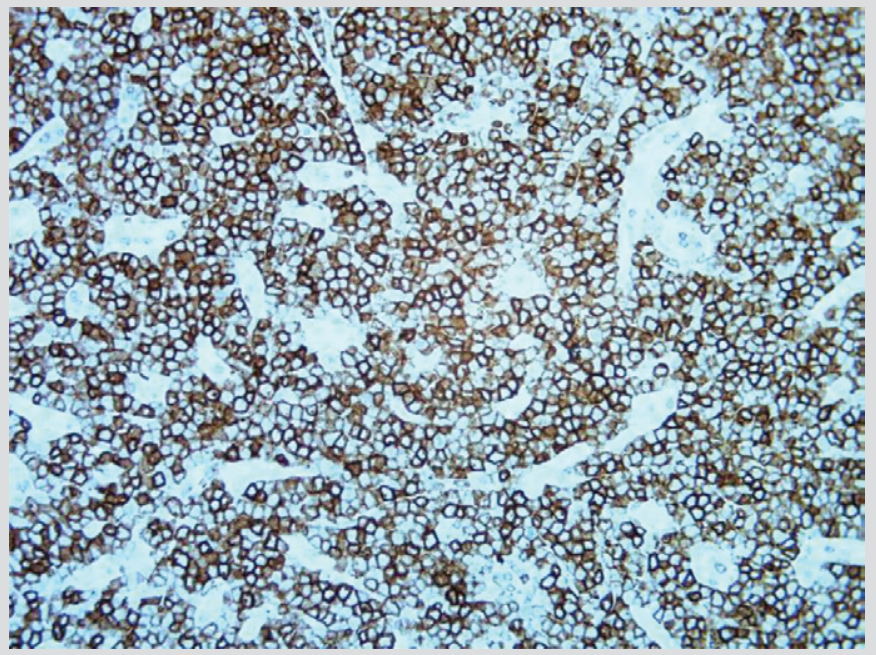

Figure 3. CD5.

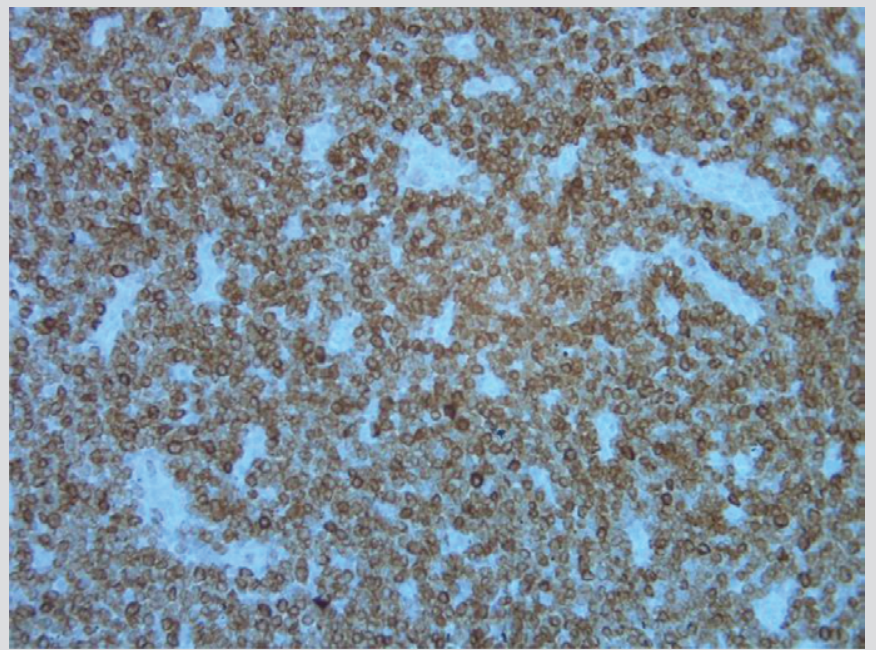

Figure 5. CD79a.

Figures 2-5. Excisional lymph node biopsy with immunohistochemistry was positive for CD3 (Figure 2), CD5 (Figure 3), CD7, TdT (Figure 4), and CD79a (Figure 5).

antigen. The tumor was positive for CD3, CD79a, and BCL-2; negative for CD5, CD20, cyclin D. The Ki-67 of this tumor was $90 \%$, indicating very high proliferation.

Three cycles of cyclophosphamide, etoposide, vincristine and prednisone (CEOP regimen) were given. Doxorubicin was initially omitted due to his heart failure. After chemotherapy, the patient's shortness of breath and functional status improved. Bone marrow biopsy was performed and showed a normocellular marrow with active hematopoiesis. Due to palpable lymph nodes in the neck and axillae, CT scans of the chest, abdomen, and pelvis were ordered and revealed increasing lymphadenopathy in the supraclavicular, mediastinal, hilar, axillary, and retroperitoneal areas and an enlarging nodule in lateral right chest suggesting progression of the disease. Due to progression of the disease with CEOP regimen, he was advanced to HyperCVAD regimen (cyclophosphamide, vincristine, doxorubicin, 
dexamethasone, and intrathecal methotrexate) for 8 cycles. He tolerated chemotherapy well without major complications. Repeat CT scans of the chest, abdomen, and pelvis reported a decrease in size of anterior mediastinal, right hilar, and retroperitoneal adenopathy. Echocardiographic examinations were done throughout the chemotherapy period. Ejection fraction with strain rate imaging was used to assess left ventricular function. The patient was placed on optimal heart failure medications as tolerated to prevent chemotherapy-related cardiotoxicity. Transient depression of ejection fraction was noted during chemotherapy. The patient continued to follow with hematology and oncology outpatient until he was lost to follow-up.

\section{Discussion}

Pericardial effusion can be caused by various diseases, including viral infection, tuberculosis, autoimmune disorders, myocardial infarction, uremia, and malignancy. Our patient presented with pericardial effusion and painless adenopathy without the classic B-symptoms of fever, night sweats, and weight loss associated with lymphoma. His blood test was positive for the Coxsackie virus $B$ antibody. Coxsackie virus is one of the most common causes of pericardial effusion secondary to pericarditis and myocarditis. Initially, this evidence supported a diagnosis of infection over malignancy and led to a delay in imaging until his $3^{\text {rd }}$ admission.

This patient had a rare presentation of pericardial effusion in T-cell lymphoblastic lymphoma. Although a mediastinal mass is not uncommon in T-cell lymphoblastic lymphoma, pericardial effusion secondary to a lymphomatous mediastinal mass usually occurs later in the course of the disease. Malignant lymphoma involves the pericardium by retrograde lymphatic spread, hematogenous spread, and direct extension from other intrathoracic tumor masses. ${ }^{7}$ Median onset of cardiac presentation is 20 months after initial diagnosis. ${ }^{8}$ In our patient, pericardial fluid was negative for neoplastic cells, and pericardial tissue showed fibrosis with reactive lymphohistiocyte infiltrate. Without immunohistochemistry stain and flow cytometry, reactive lymphoid cells may be difficult to distinguish from malignant cells. 5,6,9 T-cell lymphoblastic lymphoma can be difficult to diagnose, especially if tissue is not derived from the lymph node where T-cells originate.

Hematolymphoid neoplasms presenting as body cavity effusions are uncommon, but occasionally can present as the first manifestation of the disease. Diffuse large B-cell lymphoma is the most common lymphoma type that presents as effusion, followed by peripheral T-cell lymphoma. ${ }^{10}$ Pleural effusion is the most frequent presentation $(75 \%)$, followed by peritoneal effusion (20.8\%), and then pericardial effusion (4.1\%). The presence of body cavity effusions, such as pericardial effusion, in patients with hematolymphoid neoplasms is associated with poor patient outcomes and also predicts disease relapse after chemotherapy. ${ }^{10} \mathrm{~A}$ detailed evaluation to detect occult malignancy should generally be performed in patients who have persistent pericarditis who are unresponsive to anti-inflammatory therapy and in those who present with a new large pericardial effusion or cardiac tamponade. ${ }^{11}$ In one case report of pericardial constriction due to malignant T-cell lymphoma, chemotherapy successfully eradicated malignant cells in lymph nodes and pericardium, but the patient did not undergo pericardiectomy and died from persistent right-sided heart failure. ${ }^{12}$ Although chemotherapy can be effective in treating malignant lymphoma, once pericardial constriction develops, pericardiectomy is usually necessary to alleviate right-sided heart failure as in this case.

Article citation: Rao A, Chariyawong P, Nantsupawat T, Warraich I, Nair N. Pericardial effusion and constriction as an initial presentation of acute t-cell lymphoblastic lymphoma. The Southwest Respiratory and Critical Care Chronicles 2019;7(28):28-32

From: The Departments of Internal Medicine (AR, PC, TN, NN) and Pathology (IW) at Texas Tech University Health Sciences Center in Lubbock, Texas

Submitted: $1 / 5 / 2019$

Accepted: 4/2/2019

Reviewers: Catherine Jones MD, Menfil OrellanaBarrios MD

Conflicts of interest: none

This work is licensed under a Creative Commons Attribution-ShareAlike 4.0 International License. 


\section{REFERENCES}

1. Han X, Kilfoy B, Zheng T, et al. Lymphoma survival patterns by WHO subtype in the United States, 1973-2003. Cancer Causes Control 2008 Oct;19(8):841-58.

2. He XL, Yu F, Guo T, et al. T-cell lymphoblastic lymphoma presenting with pleural effusion: A case report. Respir Med Case Rep. 2014 May 4;12:55-8.

3. Dores GM, Devesa SS, Curtis RE, et al. Acute leukemia incidence and patient survival among children and adults in the United States, 2001-2007. Blood 2012 Jan 5;119(1): 34-43.

4. Muthusamy P, Ebrom S, Cohle SD, et al. Pericardial involvement as an initial presentation of anaplastic large cell lymphoma. Can Fam Physician 2014 Jul;60(7):638-41.

5. El Sayed MJ, Gharzuddin W, Kharfan-Dabaja MA. T-cell acute lymphoblastic leukemia presenting as a cardiac tamponade. Austin J Emergency \& Crit Care Med 2014; 1(2):4.
6. Martin SE, Zhang HZ, Magyarosy E, et al. Immunologic methods in cytology: definitive diagnosis of non-Hodgkin's lymphomas using immunologic markers for T- and B-cells. Am J Clin Pathol 1984 Dec;82(6):666-73.

7. McDonnell PJ, Mann RB, Bulkley BH. Involvement of the heart by malignant lymphoma: a clinicopathologic study. Cancer 1982 Mar 1;49(5):944-51.

8. Petersen CD, Robinson WA, Kurnick JE. Involvement of the heart and pericardium in the malignant lymphomas. $\mathrm{Am} \mathrm{J}$ Med Sci. 1976 Sep-Oct;272(2):161-5.

9. Chaves FP, Quillen K, Xu D. Pericardial effusion: a rare presentation of adult t-cell leukemia/lymphoma. American Journal of Hematology 77:381-383 (2004).

10. Imazio M, Demichelis B, Parrini I, et al. Relation of acute pericardial disease to malignancy. Am J Cardiol 2005;95:1393.

11. Ako J, Eto M, Kim S, et al. Pericardial constriction due to malignant lymphoma. Jpn Heart J 2000;41:673-679.

12. Monappa V, Reddy S, Kudva R. Hematolymphoid neoplasms in effusion cytology. Cytojournal 2018;15:15. 